\title{
VOLTERRA SPACES REVISITED
}

\author{
JILING $\mathrm{CAO}^{\curvearrowleft}$ and DAVID GAULD
}

(Received 6 August 2003; revised 19 March 2004)

\author{
Communicated by A. J. Pryde
}

\begin{abstract}
In this paper, we investigate Volterra spaces and relevant topological properties. New characterizations of weakly Volterra spaces are provided. An analogy of the Banach category theorem in terms of Volterra properties is obtained. It is shown that every weakly Volterra homogeneous space is Volterra, and there are metrizable Baire spaces whose hyperspaces of nonempty compact subsets endowed with the Vietoris topology are not weakly Volterra.
\end{abstract}

2000 Mathematics subject classification: primary 26A15, 54C05, 54E52.

Keywords and phrases: Baire space, dense $G_{\delta}$-set, the Vietoris topology, Volterra space, weakly Volterra space.

\section{Introduction}

Let $f: X \rightarrow Y$ be a function from one topological space $X$ into another topological space $Y$. We shall denote by $C(f)$ (respectively $D(f)$ ) the set of points at which $f$ is continuous (respectively discontinuous). Recall that $f$ is said to be pointwise discontinuous, abbreviated as $P W D$, if $C(f)$ is dense in $X$. This class of functions was originally introduced by Hankel [8] in 1870, and used to be the main object of studies in the classical real function theory until the appearance of the works of Lebesgue. It can be shown that a function of a Baire space to a metric space is PWD if and only if $D(f)$ is of first category. In 1881, Volterra [16] proved the following interesting theorem.

THEOREM 1.1 ([16]). Let $f: \mathbb{R} \rightarrow \mathbb{R}$ be a PWD function. Then there exists no other $P W D$ function $g: \mathbb{R} \rightarrow \mathbb{B}$ with $C(g)=D(f)$.

The first author was supported by a fellowship from the Foundation for Research, Science and Technology of New Zealand under the project number UOAX0240.

(C) 2005 Australian Mathematical Society $1446-7887 / 05 \$ A 2.00+0.00$ 
Hence, for example, the set $C(f)$ of the function $f: \mathbb{R} \rightarrow \mathbb{R}$ given by

$$
f(x)=\sum_{k \geq 1} \frac{(k x)}{k^{2}}=\frac{(x)}{1^{2}}+\frac{(2 x)}{2^{2}}+\cdots+\frac{(k x)}{k^{2}}+\cdots,
$$

where $(x)$ denotes the fractional part of $x \in \mathbb{R}$, is precisely the irrationals, and there exists no function $g: \mathbb{R} \rightarrow \mathbb{R}$ whose set of points of continuity is the rationals. These ideas and their generalizations have been studied in the last ten years by Gauld, Greenwood and Piotrowski in [3-6] respectively. Their work leads to the following definitions of Volterra and weakly Volterra spaces.

DEFINITION 1.2 ([5]). A topological space $X$ is called Volterra (respectively weakly Volterra) if for each pair of real-valued PWD functions $f, g: X \rightarrow \mathbb{R}$, the set $C(f) \cap C(g)$ is dense (respectively nonempty) in $X$.

We notice that the range space $\mathbb{R}$ in Definition 1.2 can be replaced by any developable space by considering the generalized oscillation. Although Volterra and weakly Volterra spaces are defined in terms of 'external' functions on them, there are some 'internal' characterizations for these two classes of spaces as well, namely, a space $X$ is Volterra (respectively weakly Volterra) if and only if the intersection of any two dense $G_{\delta}$-sets in $X$ is dense (respectively nonempty) [6]. Recall that a space is Baire (respectively of second category) if the intersection of any countably many dense open subsets is dense (respectively nonempty). Now, it is clear that every Baire space is Volterra, and every space of second category is weakly Volterra. Of course, all nonempty Baire spaces are of second category, and all nonempty Volterra spaces are weakly Volterra. In general, these four classes of spaces are all distinct, and relevant examples can be found in [4-7]. In answering a question in [4], Gruenhage and Lutzer [7] provided some natural classes of topological spaces in which a space is Volterra if and only if it is Baire. In particular, the following theorem is essentially proved in [7].

THEOREM 1.3 ([7]). Let $X$ be a topological space which satisfies any one of the following conditions:

(a) X contains a dense metrizable subspace.

(b) $X$ is a Lašnev space, that is, a closed continuous image of a metric space.

(c) $X$ is a metacompact sequential space which has a $\sigma$-closed discrete dense set.

(d) $X$ is separable and sequential.

(e) $X$ is a metacompact Moore space.

Then $X$ is a Baire space (respectively a space of second category) if and only if it is a Volterra (respectively weakly Volterra) space.

However, it is still not clear how to extend Theorem 1.3 to some classes of topological spaces with certain types of generalized metric properties. For example, it is 
still an open question whether it is true that every Volterra Moore space is Baire, see, for example, [7, Question 2.11].

In this paper, we shall continue the study of Volterra and weakly Volterra spaces. In Section 2, new characterizations of weakly Volterra spaces are given, and an error in a result of [4] is corrected. In Section 3, an analogy of the Banach category theorem is established. This enables us to discover a decomposition for an arbitrary topological space in terms of Volterra properties, and further prove that any weakly Volterra homogeneous space is Volterra. In the last section, we study hyperspaces of Volterra spaces with the Vietoris topology. It is shown that in certain classes of spaces, if the hyperspace of nonempty compact subsets of a given space is Volterra (respectively weakly Volterra) then all its finite powers must be Volterra (respectively weakly Volterra). We also give two examples to show that in general, the property of being (weakly) Volterra is not preserved by the hyperspace of nonempty compact subsets of a given space. Finally, some open questions related to Volterra properties of hyperspaces are posed.

All topological spaces are assumed $T_{1}$, although it is not always necessary. As usual, $\vec{A}$ and int $A$ will denote the closure and interior of a subset $A$ in a space $X$ respectively. When $X$ is a subspace of a topological space $Y$, we shall use $\bar{A}^{X}$ and int $_{X} A$ to denote the closure and interior of a subset $A$ in the subspace $X$ respectively. For a cardinal $\kappa, \operatorname{cf}(\kappa)$ denotes the cofinality of $\kappa$, and $\kappa^{+}$will represent the next cardinal after $\kappa$. The symbol ${ }^{A} B$ stands for the set of all functions from a set $A$ to a set $B$. We refer the readers to [9] for basic facts and undefined notation about Baire spaces. For the other undefined terminology, see $[11,12]$.

\section{Weakly Volterra spaces}

In this section, we first correct an error in an example of Gauld, Greenwood and Piotrowski on weakly Volterra spaces in [4]. Then, we provide some new characterizations for weakly Volterra spaces, which enable us to resolve a problem in [4]. The following result can be found in [4].

THEOREM 2.1 ([4]). If $X$ is a Volterra space, $Y_{1}, \ldots, Y_{n}(n \in \mathbb{N})$ are developable spaces and $f_{i}: X \rightarrow Y_{i}(i \leq n)$ are PWD functions, then $\bigcap\left\{C\left(f_{i}\right): 1 \leq i \leq n\right\}$ is dense in $X$.

In the light of Theorem 2.1, it is natural and also interesting to consider the following question.

QUESTION 2.2. Is it true that for any weakly Volterra space $X$, any developable spaces $Y_{1}, \ldots, Y_{n}(n \geq 3)$ and any PWD functions $f_{i}: X \rightarrow Y_{i}(1 \leq i \leq n)$, $\bigcap\left\{C\left(f_{i}\right): 1 \leq i \leq n\right\} \neq \varnothing$ ? 

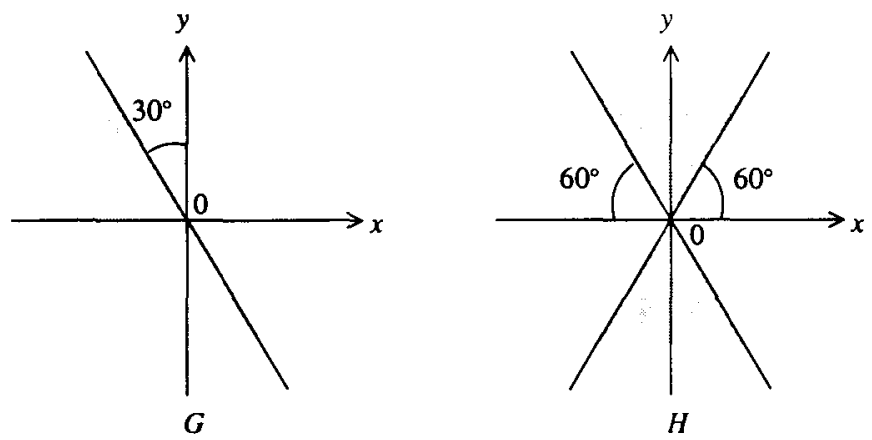

FIGURE 1 .

In fact, this question has been already considered in [4] and a negative answer was provided there. More precisely, a weakly Volterra space $X$ and three real-valued functions $f, g, h: X \rightarrow \mathbb{R}$ such that $C(f), C(g)$ and $C(h)$ are dense $G_{\delta}$-sets of $X$, but $C(f) \cap C(g) \cap C(h)=\varnothing$, were constructed in [4, Example 3]. Unfortunately, this example is false as we are going to show next.

EXAMPLE 1. The space $X$ in [4, Example 3] is not weakly Volterra. First, we shall briefly describe the space presented in [4]. Let

$$
A=\left\{\langle x, y\rangle \in \mathbb{R}^{2}: y \geq 0\right\}
$$

For each real number $r \geq 0$, let $A_{r}=\left\{\langle x, y\rangle \in \mathbb{R}^{2}: y+r>0\right\}$. Define $B, B_{r}$ to be the sets obtained by rotating $A, A, 120^{\circ}$ about $\langle 0,0\rangle$ anti-clockwise, and $C, C_{r}$ by a similar rotation clockwise. Let

$$
\begin{aligned}
& D=\left(A_{0} \cap B_{0}\right) \cup\left(B_{0} \cap C_{0}\right) \cup\left(C_{0} \cap A_{0}\right) \quad \text { and } \\
& E=\left(A_{0} \backslash(B \cup C)\right) \cup\left(B_{0} \backslash(C \cup A)\right) \cup\left(C_{0} \backslash(A \cup B)\right) .
\end{aligned}
$$

Furthermore, let us define $\mathscr{B}_{1}, \mathscr{B}_{2}$ and $\mathscr{B}_{3}$ by

$$
\begin{aligned}
& \mathscr{B}_{1}=\left\{\left(A_{r} \cap B_{s} \cap C_{t} \cap D\right) \backslash F: r, s, t>0 \text { and } F \subseteq \mathbb{R}^{2} \text { is finite }\right\} \\
& \mathscr{B}_{2}=\left\{\left(A_{r} \cap B_{s} \cap C_{t}\right) \backslash F: r, s, t>0 \text { and } F \subseteq \mathbb{R}^{2} \text { is finite }\right\} \text { and } \\
& \mathscr{B}_{3}=\left\{\left(A_{r} \cap B_{s} \cap C_{t} \cap E\right) \backslash F: r, s, t>0 \text { and } F \subseteq \mathbb{B}^{2} \text { is finite }\right\}
\end{aligned}
$$

Then the space $X$ considered in [4, Example 3] is $\mathbb{B}^{2}$ endowed with the topology generated by $\bigcup\left\{\mathscr{B}_{i}: 1 \leq i \leq 3\right\}$ as a base. It is clear that $A, B, C$ are dense $G_{\delta}$-sets of $X$. In addition, it can be checked easily that both $A_{0} \cap B_{0}$ and $C \backslash(A \cup B)$ are $G_{\delta}$-sets of $X$ (but, they are not dense in $X$ ). 
Now, consider the two subsets $G$ and $H$ of $X$ shown in Figure 1 as the two shaded regions without including their boundaries. These two sets can be defined by the following formulae

$$
G=\left(A_{0} \cap B_{0}\right) \cup(C \backslash(A \cup B)) \text { and } H=\left(B_{0} \cap C_{0}\right) \cup(A \backslash(B \cup C)) .
$$

It is not difficult to see that $G$ is dense in $X$. Being the union of two $G_{\delta}$-sets in $X, G$ is also a $G_{\delta}$-set of $X$. Thus, $G$ is a dense $G_{\delta}$-set in $X$. Similarly, $H$ is also a dense $G_{\delta}$-set of $X$. However, it is obvious that $G \cap H=\varnothing$. Therefore, we have verified that the space $X$ is not weakly Volterra.

Interestingly, the answer to Question 2.2 is positive. To show this, we shall first provide some new characterizations for weakly Volterra spaces.

THEOREM 2.3. The following statements are equivalent for a space $X$ :

(a) $X$ is a weakly Volterra space.

(b) The intersection of any finitely many dense $G_{\delta}$-sets of $X$ is somewhere dense in $X$.

(c) The intersection of any finitely many dense $G_{\delta}$-sets of $X$ is not empty.

Proof. It is clear that (b) $\Rightarrow$ (c) and (c) $\Rightarrow$ (a).

We shall prove (a) $\Rightarrow$ (b) by induction. Suppose $X$ is weakly Volterra. First, for any two dense $G_{\delta}$-sets $A_{1}, A_{2}$ of $X$, we define $B_{1}=A_{1} \backslash \overline{A_{1} \cap A_{2}}$ and $B_{2}=$ $A_{2} \backslash \overline{A_{1} \cap A_{2}}$. It is obvious that $B_{1} \cap B_{2}=\varnothing$. Since $A_{1}$ and $A_{2}$ are dense in $X$, we have $\overline{B_{1}}=X \backslash$ int $\overline{A_{1} \cap A_{2}}$, and $\overline{B_{2}}=X \backslash$ int $\overline{A_{1} \cap A_{2}}$. If int $\overline{A_{1} \cap A_{2}}=\varnothing$, then $B_{1}$ and $B_{2}$ are two dense $G_{\delta}$-sets of $X$ which are disjoint. This is a contradiction. Therefore, we have shown that the intersection of any two dense $G_{\delta}$-sets of $X$ is somewhere dense in $X$.

Next, suppose that it has been shown that the intersection of any $i$ many dense $G_{\delta}$-sets of $X$ is somewhere dense in $X$, where $1 \leq i \leq n$ and $n \geq 3$. Let $A_{1}, \ldots, A_{n+1}$ be $n+1$ many dense $G_{\delta}$-sets of $X$. Then, by our induction hypothesis, int $\overline{\bigcap\left\{A_{i}: 1 \leq i \leq n\right\}} \neq \varnothing$. For each $1 \leq j \leq n-1$, let us define the subset $C_{j} \subset X$ by

$$
C_{j}=\left(A_{j} \backslash \overline{\bigcap\left\{A_{i}: 1 \leq i \leq n\right\}}\right) \cup\left(\bigcap\left\{A_{i}: 1 \leq i \leq n\right\}\right) .
$$

Furthermore, we define the set $C_{n} \subset X$ by the following

$$
C_{n}=\left(A_{n} \backslash \overline{\bigcap\left\{A_{i}: 1 \leq i \leq n\right\}}\right) \cup\left(A_{n+1} \cap \operatorname{int} \overline{\bigcap\left\{A_{i}: 1 \leq i \leq n\right\}}\right) .
$$


Now for every $1 \leq j \leq n-1$, since $A_{j}$ is dense in $X$, we have

$$
\begin{aligned}
\overline{C_{j}} & =\bar{X} \overline{\widehat{\bigcap\left\{A_{i}: 1 \leq i \leq n\right\}}} \cup \overline{\bigcap\left\{A_{i}: 1 \leq i \leq n\right\}} \\
& =\left(X \backslash \operatorname{int} \overline{\bigcap\left\{A_{i}: 1 \leq i \leq n\right\}}\right) \cup \overline{\bigcap\left\{A_{i}: 1 \leq i \leq n\right\}} \\
& =X
\end{aligned}
$$

Thus, all the sets $C_{j}(1 \leq j \leq n-1)$ are dense $G_{\delta}$-sets of $X$. Similarly, one can check $C_{n}$ is also a dense $G_{\delta}$-set in $X$. Moreover, it is easy to see that

$$
\bigcap\left\{C_{j}: 1 \leq j \leq n\right\} \subset \bigcap\left\{A_{i}: 1 \leq i \leq n+1\right)
$$

By our induction hypothesis again, $\bigcap\left\{C_{j}: 1 \leq j \leq n\right\}$ is somewhere dense in $X$, then so is $\bigcap\left\{A_{i}: 1 \leq i \leq n+1\right\}$.

Our next result shall provide an affirmative answer to Question 2.2.

COROLlARY 2.4. Let $X$ be a weakly Volterra space, $Y_{1}, \ldots, Y_{n}(n \in \mathbb{N})$ developable spaces and $f_{i}: X \rightarrow Y_{i}(1 \leq i \leq n) P W D$ functions. Then $\bigcap_{i=1}^{n}\left\{C\left(f_{i}\right)\right\} \neq \varnothing$.

PROOF. It is easy to see that each $C\left(f_{i}\right)(1 \leq i \leq n)$ is a dense $G_{\delta}$-set of $X$. Hence, by Theorem 2.3 , we obtain $\bigcap\left\{C\left(f_{i}\right): 1 \leq i \leq n\right\} \neq \varnothing$.

\section{Volterraness in homogeneous spaces}

A space $X$ is said to be homogeneous if for any two distinct points $x, y \in X$ there exists a homeomorphism $f: X \rightarrow X$ such that $f(x)=y$. In this section, the following main theorem shall be proved.

THEOREM 3.1. Let $X$ be a homogeneous space. Then $X$ is Volterra if and only if it is weakly Volterra.

To achieve this goal, we shall first study non-weakly Volterra subspaces in a given space. It is shown that the rôle of non-weakly Volterra subspaces in the theory of Volterra spaces is somehow similar to that of first category sets in the theory of Baire spaces. In what follows, we split the proof of Theorem 3.1 into several lemmas, which are interesting for their own sake.

LEMMA 3.2. If a space $X$ contains a nonempty weakly Volterra open subspace $Y$, then $X$ itself is weakly Volterra. 
Proof. Suppose that $U$ and $V$ are any two dense $G_{\delta}$-sets in $X$. Then $U \cap Y$ and $V \cap Y$ are two dense $G_{\delta}$-sets in the subspace $Y$. Since $Y$ is weakly Volterra, then $U \cap V \supset(U \cap V) \cap Y \neq \varnothing$. Hence, $X$ is weakly Volterra.

REMARK. In Lemma 3.2, ' $Y$ is open' can be replaced with a weaker condition 'there exists a $G_{\delta}$-set $H$ in $Y$ such that int $H$ is dense in $Y$ '.

LEMMA 3.3 ([5]). A space is Volterra if and only if every nonempty open subspace is weakly Volterra.

LEMMA 3.4. If a space $X$ contains a dense $G_{\delta}$-subspace that is not weakly Volterra, then $X$ itself is not weakly Volterra.

Proof. Let $Y \subset X$ be a dense $G_{\delta}$-subspace that is not weakly Volterra. Then there are two disjoint dense $G_{\delta}$-sets $U$ and $V$ in $Y$. Pick two dense $G_{\delta}$-sets $\hat{U}$ and $\hat{V}$ in $X$ with $U=\hat{U} \cap Y$ and $V=\hat{V} \cap Y$. Suppose that $X$ is weakly Volterra. Then, by Theorem 2.3 (c), we have $\hat{U} \cap \hat{V} \cap Y \neq \varnothing$. It follows that $U \cap V \neq \varnothing$. This is a contradiction, since $U \cap V=\varnothing$.

Since every non-weakly Volterra subspace in a topological space must be a set of first category, our next lemma can be treated as an analogy of the Banach category theorem in topology and analysis.

LEMMA 3.5. In any space $X$, the union of any family of nonempty open non-weakly Volterra subspaces is not weakly Volterra.

PROOF. Let $\mathscr{U}$ be a family of nonempty open subspaces of $X$ such that each member of $\mathscr{U}$ is not weakly Volterra in $X$. Let $\Im_{N V}$ be the set of all collections of nonempty open subsets of $X$ with the following two properties:

(a) each collection $\mathscr{V} \in \Im_{N V}$ is pairwise disjoint; and

(b) for each collection $\mathscr{V} \in \Im_{N V}$ and each member $V \in \mathscr{V}$, there exists some $U \in \mathscr{U}$ such that $V \subset U$.

Then, by Zorn's lemma, $\Im_{N V}$ has a maximal element $\mathscr{V}=\left\{V_{\alpha}: \alpha \in A\right\}$. Let $V=\bigcup\left\{V_{\alpha}: \alpha \in A\right\}$. By the maximality of $\mathscr{V}$, we have $\bigcup\{U: U \in \mathscr{U}\} \subset \bar{V}$. Moreover, it follows from (b) and Lemma 3.2 that for each $\alpha \in A, V_{\alpha}$ is not weakly Volterra as an open subspace of $X$. Thus, there are two families $\left\{F_{\alpha}: \alpha \in A\right\}$ and $\left\{H_{\alpha}: \alpha \in A\right\}$ of $G_{\delta}$-sets of $X$ such that

(c) $F_{\alpha} \cap H_{\alpha}=\varnothing$ for all $\alpha \in A$; and

(d) $F_{\alpha} \subset V_{\alpha} \subset \overline{F_{\alpha}}$ and $H_{\alpha} \subset V_{\alpha} \subset \overline{H_{\alpha}}$ for all $\alpha \in A$. 
Let $F=\bigcup\left\{F_{\alpha}: \alpha \in A\right\}$ and $H=\bigcup\left\{H_{\alpha}: \alpha \in A\right\}$. By (a) and (c), we have $F \cap H=\varnothing$. For each $\alpha \in A$, let

$$
F_{\alpha}=\bigcap\left\{F_{\alpha}^{n}: n \geq 1\right\} \text { and } H_{\alpha}=\bigcap\left\{H_{\alpha}^{n}: n \geq 1\right\},
$$

where $F_{\alpha}^{n}$ and $H_{\alpha}^{n}$ are nonempty open subsets of $X$ contained in $V_{\alpha}$ such that $F_{\alpha}^{n+1} \subset F_{\alpha}^{n}$ and $H_{\alpha}^{n+1} \subset H_{\alpha}^{n}$ for all $\alpha \in A$ and all $n \in \mathbb{N}$. Now, put

$$
F_{n}=\bigcup\left\{F_{\alpha}^{n}: \alpha \in A\right\} \quad \text { and } H_{n}=\bigcup\left\{H_{\alpha}^{n}: \alpha \in A\right\} .
$$

for all $n \in \mathbb{N}$. After a simple computation, we can obtain

$$
F=\bigcap\left\{F_{n}: n \geq 1\right\} \text { and } H=\bigcap\left\{H_{n}: n \geq 1\right\} .
$$

By (d), we have $V_{\alpha} \subset \bar{F}_{\alpha} v$ and $V_{\alpha} \subset \bar{H}_{\alpha} v$ for each $\alpha \in A$. Since $\left\{F_{\alpha}: \alpha \in A\right\}$ and $\left\{H_{\alpha}: \alpha \in A\right\}$ are two discrete families in the subspace $V$ of $X$, then

$$
V \subset \bigcup\left\{\bar{F}_{\alpha}^{v}: \alpha \in A\right\}=\bar{F}^{V} \text { and } \quad V \subset \bigcup\left\{\bar{H}_{\alpha}^{v}: \alpha \in A\right\}=\bar{H}^{v} .
$$

Thus, $F$ and $H$ are two disjoint dense $G_{\delta}$-sets in the subspace $V$ of $X$. Consequently, $V$ is not a weakly Volterra subspace of $X$. It follows from Lemma 3.4 that $\bar{V}$ is not a weakly Volterra subspace of $X$ either. Since $\bigcup\{U: U \in \mathscr{U}\} \subset \bar{V}$, by Lemma 3.2 again, $\bigcup\{U: U \in \mathscr{U}\}$ is not a weakly Volterra subspace of $\bar{V}$. Therefore, we conclude that $\bigcup\{U: U \in \mathscr{U}\}$ is not a weakly Volterra subspace of $X$.

As an immediate application of Lemma 3.5, we obtain the following decomposition lemma for an arbitrary topological space.

LemMA 3.6. Let $X$ be an arbitrary topological space. Then there are two open (possibly empty) subspaces $X_{N V}$ and $X_{V}$ of $X$ such that

(a) $X=\overline{X_{N V}} \cup \overline{X_{V}}$ and $X_{N V} \cap X_{V}=\varnothing$;

(b) every nonempty open subspace of $X_{N V}$ is not weakly Volterra in $X$; and

(c) every nonempty open subspace of $X_{V}$ is Volterra in $X$.

Furthermore, $X$ is a Volterra space if and only $X_{N V}=\varnothing$, and $X$ is a weakly Volterra space if and only if $X_{V} \neq \varnothing$.

PROOF. Let $X_{N V}$ be the union of all nonempty open non-weakly Volterra subspaces of $X$, and let $X_{V}=X \backslash \overline{X_{N V}}$. By Lemma 3.5, $X_{N V}$ is not weakly Volterra as an open subspace of $X$. It is obvious that every nonempty open subspace of $X_{V}$ is weakly Volterra. Thus, following from Lemma 3.3, every nonempty open subspace of $X_{V}$ is Volterra. So, we have shown that $X_{N V}$ and $X_{V}$ fulfil (a), (b) and (c). By Lemma 3.3 again, $X$ is Volterra if and only if $X_{N V}=\varnothing$. If $X V \neq \varnothing$, then $X_{V}$ is a weakly Volterra 
subspace of $X$. By Lemma 3.2, the space $X$ itself is weakly Volterra. Conversely, suppose that $X$ is weakly Volterra, and $X_{V}=\varnothing$. Then $X=\overline{X_{N V}}$. Since $X_{N V}$ is not weakly Volterra, then by Lemma 3.4, the space $X$ itself is not weakly Volterra either. This is a contradiction.

Now we are able to prove Theorem 3.1 by applying the previous lemmas.

Proof of TheOREM 3.1. The necessity is trivial. To prove the sufficiency, suppose that $X$ is a weakly Volterra space. Then, by Lemma 3.6, $X_{V}$ is a nonempty open Volterra subspace of $X$. Now, let $U$ be any nonempty open subspace of $X$. Then there exists a point $x \in X_{V}$ and a homeomorphism $f: X \rightarrow X$ such that $f(x) \in U$. The space $U \cap f\left(X_{V}\right)$, being a nonempty open subspace of the Volterra space $f\left(X_{V}\right)$, is also Volterra. Thus, it follows from Lemma 3.2 that $U$ is a weakly Volterra subspace of $X$. Finally, by Lemma 3.3, the space $X$ itself is Volterra.

The relationships among the classes of Baire spaces, Volterra spaces, weakly Volterra spaces and spaces of second category can be summarised in the following figure.

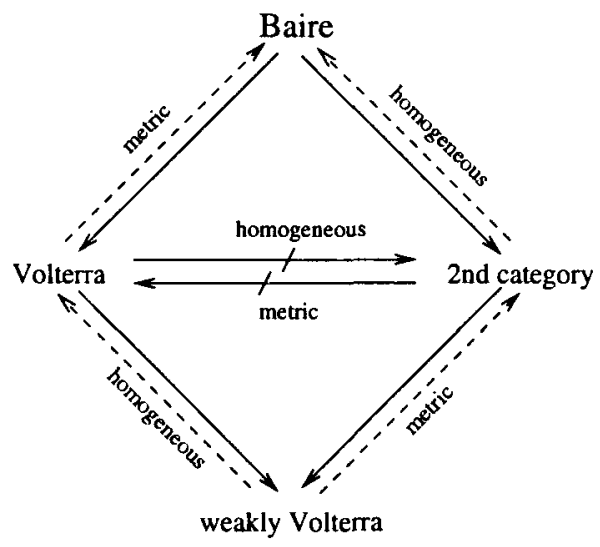

FIGURE 2.

REMARK. It is well known that a homogeneous space is Baire if and only if it is of second category. Note that homogeneous Volterra spaces which are not Baire do exist. For example, let $X=\mathbb{R}$ be the set of all reals. Let $\mathscr{T}_{1}$ be the lower topology on $X$, that is, $\mathscr{T}_{1}=\{\varnothing, X\} \cup\{(a,+\infty): a \in X\}$. Let $\mathscr{T}_{2}$ be the co-countable topology on $X$. Equip $X$ with the topology $\mathscr{T}=\mathscr{T}_{1} \vee \mathscr{T}_{2}$. Then $X$ is a $T_{1}$ homogeneous space. Every dense $G_{\delta}$-set $A$ of $X$ can be expressed by either $A=X \backslash S$, or $A=(a,+\infty) \backslash S$, or $A=[a,+\infty) \backslash S$, where $a \in X$ and $S \subset X$ is countable. Hence, the intersection of any finitely many dense $G_{\delta}$-sets of $X$ meets every nonempty member of $\mathscr{T}$. It 
follows that $X$ is Volterra. On the other hand, $X$ is not Baire, because the subsets $U_{n}=(n,+\infty)$ of $X$ are all open and dense but their intersection over $\mathbb{N}$ is empty.

Since the space given in the previous remark is not Hausdorff, the following question arises naturally.

QUESTION 3.7. Does there exist a Tychonoff homogeneous space or even a Hausdorff topological group which is Volterra but not Baire?

By Lemma 3.6, a nonempty space is not weakly Volterra if and only if no nonempty open subspace is weakly Volterra. Our next result, which says that a semi-open subspace of a given space is not weakly Volterra if and only if it is nowhere weakly Volterra, is a slight extension of this fact. Recall that a subset $A$ of a space $X$ is semi-open if int $A$ is dense in $A$. It is clear that in any topological space, all open subspaces are semi-open.

THEOREM 3.8. Let $A$ be a nonempty semi-open subspace of a space $X$. Then $A$ is not weakly Volterra in $X$ if and only if for every open subset $U$ of $X$ with $U \cap A \neq \varnothing$ there exists a nonempty open subset $V$ of $X$ contained in $U$ such that $V \cap A$ is not weakly Volterra in $X$.

ProOF. The necessity follows from Lemma 3.2 directly. So, we shall consider the sufficiency. First, suppose that $A$ is a nowhere dense subset of $X$. Let $U$ and $V$ be any two dense $G_{\delta}$-sets in $A$. If $A$ is weakly Volterra, then by Theorem $2.3, U \cap V$ is a somewhere dense set in the subspace $A$. We shall derive a contradiction. Let $G$ be any nonempty open subset of $A$, and let $H$ be an open subset of $X$ with $G=H \cap A$. Then $H \cap \operatorname{int} A \neq \varnothing$, as int $A$ is dense in $A$. Since $A$ is a nowhere dense set of $X$, then $U \cap V$ is a nowhere dense subset of $X$ as well. Thus, there exists a nonempty open subset $O$ of $X$ contained in $H \cap$ int $A$ such that $O \cap(U \cap V)=\varnothing$. This shows that $U \cap V$ is a nowhere dense set in the subspace $A$, which is a contradiction. Hence, $A$ is not weakly Volterra in this case.

Next, we shall consider the case that $A$ is a somewhere dense subset of $X$. Let $U=\operatorname{int} \bar{A}$. Then $U$ is a nonempty open subset of $X$. Let $\mathscr{U}=\left\{U_{\beta}: \beta \in B\right\}$ be the family of all nonempty open subsets of $X$ such that for each $\beta \in B, U_{\beta} \subset U$ and $U_{\beta} \cap A$ is not a weakly Volterra subspace of $X$. Note that for each $\beta \in B, U_{\beta} \cap A$ is not weakly Volterra as an open subspace of the subspace $A$. It follows from Lemma 3.5 that $\bigcup\left\{U_{\beta} \cap A: \beta \in B\right\}$ is not weakly Volterra in the subspace $A$. By hypothesis, $\bigcup\left\{U_{\beta} \cap A: \beta \in B\right\}$ is a dense open subspace of $A \cap U$. Hence, it follows from Lemma 3.4 that $A \cap U$ cannot be weakly Volterra. Furthermore, since int $A \subset A \cap U$, by Lemma 3.2, int $A$ is not weakly Volterra in $X$. Finally, as int $A$ is dense and open in the subspace $A$, by Lemma 3.4 again, we conclude that $A$ is not a weakly Volterra subspace of $X$. 
REMARK. Note that the condition ' $A$ is semi-open' in Theorem 3.8 is not needed in the proof of necessity. However, the authors do not know whether this condition can be dropped from the proof of the sufficiency.

\section{Hyperspaces of Volterra spaces}

In this section, we shall study hyperspaces of Volterra and weakly Volterra spaces. For a given Hausdorff topological space $X$, let $2^{X}$ denote the collection of nonempty closed subsets of $X$. For any finite family $\mathscr{U}=\left\{U_{1}, U_{2}, \ldots, U_{n}\right\}$ of subsets of $X$, we define $\langle\mathscr{U}\rangle \subset 2^{X}$ by

$$
\langle\mathscr{U}\rangle=\left\{F \in 2^{X}: F \subset \bigcup\left\{U_{i}: 1 \leq i \leq n\right\}, \text { and } F \cap U_{i} \neq \varnothing \forall i=1, \ldots, n\right\} .
$$

Throughout this section, $2^{X}$ shall be equipped with the so-called Vietoris topology $\tau_{V}$ (also known as the finite topology in the literature), which has the family of all subsets of $2^{X}$ of the form $\langle\mathscr{U}\rangle$ as a base, where $\mathscr{U}$ runs through all finite families of open subsets of $X$. Let $\mathscr{F}(X)$ (respectively $\mathscr{K}(X)$ ) be the subspace of $2^{X}$ consisting of all nonempty finite (respectively compact) subsets of $X$ with the relative topology. In what follows, we shall first give some necessary conditions for space $X$ in certain classes of spaces such that $\mathscr{K}(X)$ is (weakly) Volterra. Then we give two examples from well-known constructions to show that the (weak) Volterraness of a space $X$ is not preserved by its hyperspace $\mathscr{K}(X)$ in general.

LEMMA 4.1. For any Hausdorff space $X$, if $\mathscr{K}(X)$ is Volterra (respectively weakly Volterra) then $X$ is Volterra (respectively weakly Volterra).

PROOF. For a family $\left\{B_{\alpha}: \alpha \in A\right\}$ of subsets of $X$, it is easy to check that

(a) $\left\langle\bigcap\left\{B_{\alpha}: \alpha \in A\right\}\right\rangle=\bigcap\left\{\left\langle B_{\alpha}\right\rangle: \alpha \in A\right\}$; and

(b) for any $\alpha \in A, B_{\alpha}$ is dense (respectively nonempty) in $X$ if and only if $\left\langle B_{\alpha}\right\rangle$ is dense (respectively nonempty) in $\mathscr{K}(X)$.

Now suppose that $\mathscr{K}(X)$ is Volterra (respectively weakly Volterra). Let $U$ and $V$ be two dense $G_{\delta}$-sets in $X$. Then $\langle U\rangle$ and $\langle V\rangle$ are dense $G_{\delta}$-sets in $\mathscr{K}(X)$. Since $\mathscr{K}(X)$ is Volterra (respectively weakly Volterra), then $\langle U\rangle \cap\langle V\rangle$ is dense (respectively nonempty) in $\mathscr{K}(X)$. By (a) and (b) above, $U \cap V$ is dense (respectively nonempty) in $X$. Therefore, $X$ is Volterra (respectively weakly Volterra).

We notice that the conclusion of Lemma 4.1 still holds when $\mathscr{K}(X)$ is replaced by $2^{X}$. Next, we shall show that the conclusion of Lemma 4.1 can be strengthened for certain classes of spaces. 
THEOREM 4.2. Let $X$ be a Tychonoff space which satisfies any one of the following conditions:

(a) X has a dense metrizable subspace.

(b) $X$ is a Lašnev space, that is, a closed continuous image of a metric space.

(c) $X$ is separable and first countable.

(d) $X$ is a metacompact Moore space.

If $\mathscr{K}(X)$ is a Volterra (respectively weakly Volterra) space, then $X^{n}$ is a Baire space (respectively a space of second category) for all $n \in \mathbb{N}$.

Proof. Suppose that $\mathscr{K}(X)$ is a Volterra (respectively weakly Volterra) space. By Lemma 4.1, $X$ itself is Volterra (respectively weakly Volterra). Then, by Theorem 1.3, under any of these conditions, $X$ is a Baire space (respectively a space of second category). We first show that under any one of these conditions, $\mathscr{K}(X)$ is a Baire space (respectively a space of second category). The cases of (a), (b) and (c), which are easier, shall be shown in the next. Suppose that (a) holds. Let $Y \subset X$ be a dense metrizable subspace. Then $\mathscr{F}(Y)$ is a dense metrizable subspace of $\mathscr{K}(X)$. If (b) holds, then there is a metric space $M$ and a closed continuous mapping $f: M \rightarrow X$ from $M$ onto $X$. Define $\hat{f}: \mathscr{K}(M) \rightarrow \mathscr{K}(X)$ by letting $\hat{f}(K)=f(K)$ for all $K \in \mathscr{K}(M)$. It can be checked that $\hat{f}$ is closed and continuous. Moreover, $\hat{f}(\mathscr{K}(M))$ is a dense subspace of $\mathscr{K}(X)$. Now, suppose that (c) holds. Then $\mathscr{F}(X)$ is a dense separable and first countable subspace of $\mathscr{K}(X)$. Hence, by [7, Corollary 2.8], under any of conditions (a), (b) and (c), $\mathscr{K}(X)$ is Baire (respectively of second category).

Finally, suppose (d) holds. Then we can choose a development $(\mathscr{U})_{n \in \mathbb{N}}$ such that for each $n \in \mathbb{N}, \mathscr{U}_{n}$ is a point finite open cover of $X$ and $\mathscr{U}_{n+1}$ is a refinement of $\mathscr{U}_{n}$. For each $n \in \mathbb{N}$, let $Y_{n} \subset X$ be a dense $G_{\delta}$-subspace such that $\mathscr{U}_{n}$ is locally finite at each point of $Y_{n}$. For each $n \in \mathbb{N}$, set

$$
Y=\bigcap\left\{Y_{n}: n \in \mathbb{N}\right\} \text { and } \mathscr{V}_{n}=\left\{U \cap Y: U \in \mathscr{U}_{n}\right\} .
$$

Then, $\bigcup\left\{\mathscr{V}_{n}: n \in \mathbb{N}\right\}$ is a $\sigma$-locally finite base for $Y$. Thus, by the Bing-NagataSmirnov metrization theorem, $Y$ is a metrizable subspace of $X$. If $\mathscr{K}(X)$ is Volterra, as we have seen, $X$ is Baire. Then, $Y$ is dense in $X$, and thus $\mathscr{F}(Y)$ is a dense metrizable subspace of $\mathscr{K}(X)$. It follows that $\mathscr{K}(X)$ is Baire. Suppose that $\mathscr{K}(X)$ is weakly Volterra. By Lemma $3.6, \mathscr{K}(X)$ contains a nonempty basic open subspace $\left\langle U_{1}, \ldots, U_{n}\right\rangle$ that is Volterra. Let $U=\bigcup\left\{U_{i}: 1 \leq i \leq n\right\}$. We claim that $U$ is an open Volterra subspace of $X$. To see this, for any two dense $G_{\delta}$-sets

$$
G=\bigcap\left\{G_{m}: m \in \mathbb{N}\right\} \text { and } H=\bigcap\left\{H_{m}: m \in \mathbb{N}\right\}
$$

of $U$, where $G_{m}$ and $H_{m}$ are open subsets of $U$ (thus they are open in $X$ as well) for all $m \in \mathbb{N}$, let $G_{m i}=G_{m} \cap U_{i}$ and $H_{m i}=H_{m} \cap U_{i}$ for each $1 \leq i \leq n$. Then, for 
each $m \in \mathbb{N}$, we can define two basic open subsets

$$
\mathscr{G}_{m}=\left\langle G_{m 1}, \ldots, G_{m n}\right\rangle \text { and } \mathscr{H}_{m}=\left\langle H_{m 1}, \ldots, H_{m n}\right\rangle
$$

in $\mathscr{K}(X)$. It can be readily checked that $\mathscr{G}=\bigcap\left\{\mathscr{G}_{m}: m \in \mathbb{N}\right\}$ and $\mathscr{H}=\bigcap\left\{\mathscr{H}_{m}\right.$ : $m \in \mathbb{N}\}$ are dense $G_{\delta}$-sets in the subspace $\left\langle U_{1}, \ldots, U_{n}\right\rangle$. Thus, $\mathscr{G} \cap \mathscr{H}$ is dense in $\left\langle U_{1}, \ldots, U_{n}\right\rangle$. This implies that $G \cap H$ is dense in $U$. Hence, we have shown that $U$ is a Volterra subspace of $X$. Next, we choose an open subset $V \subset X$ such that $\bar{V} \subset U$ and $V_{i}=V \cap U_{i} \neq \varnothing$ for all $1 \leq i \leq n$. Being a nonempty open subspace of $\left\langle U_{1}, \ldots, U_{n}\right\rangle,\left\langle V_{1}, \ldots, V_{n}\right\rangle$ is also Volterra. By applying the condition (d) to the closed subspace $\bar{V}$ of $X$ and then repeating the previous argument, we conclude that $\bar{V}$ contains a dense metrizable subspace $M$. Then $M \cap V$ is a dense metrizable subspace of $V$. Since $\mathscr{F}(M \cap V)$ is dense in $\left(V_{1}, \ldots, V_{n}\right\rangle$, then it follows that

$$
\mathscr{F}(M \cap V) \cap\left\langle V_{1}, \ldots, V_{n}\right\rangle
$$

is a dense metrizable subspace of $\left\langle V_{1}, \ldots, V_{n}\right\rangle$. By Theorem 1.3 (a), we conclude that $\left\langle V_{1}, \ldots, V_{n}\right\rangle$ is an open Baire subspace of $\mathscr{K}(X)$. Therefore, $\mathscr{K}(X)$ is a space of second category.

To complete the proof, we need to introduce some auxiliary tools. For any finite family $\mathscr{U}=\left\{U_{1}, U_{2}, \cdots, U_{n}\right\}$ of subsets of $X$, let

$$
\mathscr{U}^{*}=\prod\left\{U_{i}: 1 \leq i \leq n\right\} \times \prod\left\{\bigcup\left\{U_{j}: 1 \leq j \leq n\right\}: i>n\right\} .
$$

Then $\mathscr{U}^{*} \subset X^{\omega}$. Let $X^{\omega}$ be equipped with a topology $\tau^{*}$ by taking

$$
\Im=\left\{\mathscr{U}^{*}: \mathscr{U} \text { is a finite family of open subsets of } X\right\}
$$

as a base. We denote the space $X^{\omega}$ with this topology by $X_{*}^{\omega}$. We have shown that under any of conditions (a)-(d), $\mathscr{K}(X)$ is Baire (respectively of second category). Then it follows from [13, Theorem 3.10] that $X_{*}^{\prime \prime}$ is also a Baire space (respectively space of second category). For any fixed $n \in \mathbb{N}$, since the canonical projection mapping $\pi: X_{*}^{\omega} \rightarrow X^{n}$, defined by $\pi\left(\left\langle x_{i}\right\rangle\right)=\left\langle x_{1}, \ldots, x_{n}\right\rangle$ for all $\left\langle x_{i}\right\rangle \in X_{*}^{\omega}$, is an open and continuous surjection, then $X^{n}$ is Baire (respectively of second category).

REMARK. (i) In general, none of the following properties: first countability, Lašnev, metacompactness and sequentiality, is preserved by the hyperspace of nonempty compact subsets of a given space. For example, the Sorgenfrey line $S$ is metacompact, but $\mathscr{K}(S)$ is not metacompact. The other relevant counterexamples can be found in $[1,14]$.

(ii) For any given space $X$, the associated space $X_{*}^{\omega}$, or a more general space $X_{*}^{\kappa}$ (where $\kappa \geq \omega$ ), has been studied in $[13,15]$ respectively. In particular, $\tau^{*}$ is called the pinched-cube topology in [15]. 
Now, we give two examples to show that the hyperspace $\mathscr{K}(X)$ of a Volterra (even a metric Baire) space $X$ does not need to be weakly Volterra, that is, the converse of Lemma 4.1 does not hold in general.

EXAMPLE 2. A Baire metric space $X$ whose square $X^{2}$ is nowhere Baire and whose hyperspace $\mathscr{K}(X)$ is not weakly Volterra. For any cardinal $\kappa>\omega$, let $C_{\omega} \kappa=\{\alpha \in \kappa: \operatorname{cf}(\alpha)=\omega\}$. For any $f \in{ }^{\omega^{\omega}} \kappa$, let $f^{*}=\sup \{f(n): n \in \omega\}$. Next, define a metric $\rho$ on ${ }^{\omega} \kappa$ by

$$
\rho(f, g)= \begin{cases}0, & \text { if } f=g ; \\ 1 / 2^{n}, & \text { if } f \neq g, \text { where } n=\min \{m \in \omega: f\lceil m \neq g\lceil m\} .\end{cases}
$$

Then the metric space $\left({ }^{\omega} \kappa, \rho\right)$ is simply denoted by $J_{\kappa}$. Let $M=J_{2} \times J_{c^{+}}$be given the product metric $d$, that is, $d\left(\left\langle x_{1}, y_{1}\right\rangle,\left\langle x_{2}, y_{2}\right\rangle\right)=\rho_{1}\left(x_{1}, x_{2}\right)+\rho_{2}\left(y_{1}, y_{2}\right)$. Now, let $\left\{A_{y}: y \in J_{2}\right\}$ be a family of pairwise disjoint stationary subsets of $C_{\omega} \mathrm{c}^{+}$. Consider the subspace $X=\left\{\langle y, f\rangle \in M: f^{*} \in A_{y}\right\}$ of $M$. It is shown in [2, Example 4] that $X$ is a Baire space, but $X^{2}$ is of first category. By Theorem $4.2, \mathscr{K}(X)$ is not weakly Volterra.

EXAMPLE 3. A hereditarily Baire metric space $X$ all of whose powers are Baire, but whose hyperspace $\mathscr{K}(X)$ is not weakly Volterra. Let $X \subset \mathbb{R}$ be a Berstein set endowed with the Euclidean topology (refer to $[10,12]$ for the existence of such a set in $\mathbb{R}$ ). It is known that $X$ is a hereditarily Baire metric space such that neither $X$ nor $\mathbb{R} \backslash X$ contains a perfect subset of $\mathbb{R}$. Moreover, it is also known that all compact subsets of $X$ are countable and $X^{\kappa}$ is a Baire space for any cardinal $\kappa$. Since $X$ is separable, it has a countable base $\mathscr{B}$. For any nonempty member $V \in \mathscr{B}$, let

$$
\mathscr{A}_{V}=\{K \in \mathscr{K}(X): K \cap V \text { contains exactly one point }\} .
$$

Suppose that $\left\langle U_{1}, \ldots, U_{n}\right\rangle$ is any basic open subset of $\mathscr{K}(X)$, where $U_{1}, \ldots, U_{n}$ are nonempty open subsets of $X$. We consider two cases.

(i) $V \cap\left(\bigcup_{i=1}^{n} U_{i}\right)=\varnothing$. In this case, we have $\mathscr{A}_{V} \cap\left\langle U_{1}, \ldots, U_{n}\right\rangle=\varnothing$.

(ii) $V \cap U_{i_{0}} \neq \varnothing$ for some $1 \leq i_{0} \leq n$. First, we can choose two disjoint nonempty open subsets $U_{i_{0}}^{1}$ and $U_{i_{0}}^{2}$ of $X$ both of which are contained in $V \cap U_{i_{0}}$. Then, it follows that $\left\langle U_{1}, \ldots, U_{i_{0}}^{1}, U_{i_{0}}^{2}, \ldots, U_{n}\right\rangle$ is a basic open subset of $\mathscr{K}(X)$ which is contained in $\left\langle U_{1}, \ldots, U_{n}\right\rangle$ such that $\mathscr{A}_{V} \cap\left\langle U_{1}, \ldots, U_{i_{0}}^{1}, U_{i_{0}}^{2}, \ldots, U_{n}\right\rangle=\varnothing$.

Thus, we have shown that $\mathscr{A}_{V}$ is a nowhere dense subset of $\mathscr{K}(X)$. Since each nonempty compact subset is not perfect, it must have an isolated point. Consequently, $\mathscr{K}(X)=\bigcup_{V \in \mathscr{B}} \mathscr{A}_{V}$ and $\mathscr{K}(X)$ is a space of first category. In addition, since $\mathscr{K}(X)$ is metrizable by the Hausdorff metric of the Euclidean metric on $X$, by Theorem 1.3, $\mathscr{K}(X)$ is not weakly Volterra. 
By a result of McCoy in [13], if $X$ is a Tychonoff space such that $\mathscr{K}(X)$ is a Baire space, then $X^{n}$ is Baire for all $n \in \mathbb{N}$. We conclude this paper with the following two questions motivated by this fact and Theorem 4.2.

QUESTION 4.3. Let $X$ be a Tychonoff space. If $\mathscr{K}(X)$ is a Volterra (respectively weakly Volterra) space, is it true that $X^{n}$ is Volterra (respectively weakly Volterra) for all $n \in \mathbb{N}$ ?

QUestion 4.4. Let $X$ be a Tychonoff space. If $2^{X}$ is a Baire (respectively Volterra, weakly Volterra), is it true that $X^{n}$ is Baire (respectively Volterra, weakly Volterra) for all $n \in \mathbb{N}$ ?

NOTE ADDED IN PROOF. In the system $[Z F C+P(\mathfrak{c})]$, there is a Hausdorff-Volterra group which is not Baire; refer to V. Malykhin, 'Extremally disconnected and nearly extremally diconnected groups', Soviet Math. Dokl. 16 (1975), 21-25. Recently, an affirmative answer to the case of Baire spaces of Question 4.4 has been given by J. Cao, S. García-Ferreira and V. Gutev in a joint paper.

\section{Acknowledgement}

Example 3 was communicated to the first-listed author by T. O. Banakh as folklore at V Iberoamerican conference on General Topology and its Applications at Lorca, Spain in June, 2003.

\section{References}

[1] M. Čoban, 'Note sur la topologie exponentielle', Fund. Math. 71 (1971), $27-41$.

[2] W. Fleissner and K. Kunen, 'Barely Baire spaces', Fund. Math. 101 (1978), 229-240.

[3] D. Gauld, 'Did the young Volterra know about Cantor?', Math. Magazine 66 (1993), 246-247.

[4] D. Gauld, S. Greenwood and Z. Piotrowski, 'On Volterra spaces II', Ann. New York Acad. Sci. 806 (1996), 169-173.

[5] —. 'On Volterra spaces III: Topological operations', Topology Proc. 23 (1998), 167-182.

[6] D. Gauld and Z. Piotrowski, 'On Volterra spaces', Far East J. Math. Sci. 1 (1993), 209-214.

[7] G. Gruenhage and D. Lutzer, 'Baire and Volterra spaces', Proc. Amer. Math. Soc. 128 (2000), 3115-3124.

[8] H. Hankel, Untersuchungen über die oscilierenden und unstetigen Funktionen (1870), Ostwalds klassiker der exacten Wissenschaften 153 (Leipzig, 1905).

[9] R. C. Haworth and R. A. McCoy, Baire spaces, Dissertationes Math. 141 (Instytut Matematyczny, Polska Akademia Nauk, 1977).

[10] E. Hewitt and K. Stromberg, Real and abstract analysis. A modern treatment of the theory of functions of a real variable (Springer, New York, 1969). 
[11] K. Kunen and J. Vaughan, Handbook of set-theoretic topology (North-Holland, Amsterdam, 1984).

[12] K. Kuratowski, Topology I (Państwowe Wydawnictwo Naukowe, Warsaw, 1966).

[13] R. A. McCoy, 'Baire spaces and hyperspaces', Pacific J. Math. 58 (1975), 133-142.

[14] T. Mizokami, 'On hyperspaces of generalized metric spaces', Topology Appl. 76 (1997), 169-173.

[15] Z. Piotrowski, A. Roslanowski and B. M. Scott, 'The pinched-cube topology', Pacific J. Math. 105 (1983), 319-413.

[16] V. Volterra, 'Alcune osservasioni sulle funzioni punteggiate discontinue', Giornale di Matematiche 19 (1881), 76-86.

Department of Mathematics

The University of Auckland

Private Bag 92019

Auckland 1

New Zealand

e-mail: jil.cao@auckland.ac.nz, d.gauld@auckland.ac.nz 